\title{
CONTROL OF VIBRATIONAL FIELD IN A CYBER-PHYSICAL VIBRATION UNIT
}

\author{
Olga P. Tomchina \\ Saint Petersburg State University of Architecture and Civil Engineering (SPSUACE) \\ 2nd Krasnoarmeiskaya St. 4, 190005, St. Petersburg, Russia \\ E-mail: otomchina@mail.ru
}

Article history:

Received 04.11.2018, Accepted 28.11.2018

\begin{abstract}
The problem of feedback control of vibrational fields in a vibration unit is posed. The algorithms for control of the vibration fields by means of the control of the phase shift between rotors are proposed. The performance of the closed loop system is examined by simulation for the two-rotor vibration unit SV-2M.
\end{abstract}

\section{Key words}

Control of vibrations, vibration fields, vibration units.

\section{Introduction}

A number of interesting physical phenomena can be found in vibration units (VU). VU are used in the mining and manufacturing industries for the purpose of transporting and processing of materials or products: grinding, screening, mixing, compacting, etc. [Chelomey, 1978-1981]. The most effective equipment for vibrational transportation and separation of bulk materials by fractions are vibrating screens that have as their working body a moving screening surface (platform) [Blekhman, Vaisberg, and Firsova, 2001; Firsova, 2002(1); Firsova, 2002(2)]. The VU is usually equipped with electromechanical vibration actuators made on the basis of unbalanced rotors (debalances) driven by electric drives. Rapid development of mechatronics and cyber-physical paradigm may reveal new phenomena and pose new problems in the application of vibration units [Blekhman and Fradkov, Eds, 2001]. This paper is devoted to one of them: control of vibrational fields.

Since different points of a vibrating body oscillate along different trajectories, one may speak about vibrational field [Chelomey, 1978-1981]. A systematic approach to analysis and synthesis of the vibrational fields for vibrating units was proposed by I.I.Blekhman with coauthors in 2001-2003 [Blekhman, Vaisberg, and Firsova, 2001; Firsova, 2002(1); Firsova, 2002(2)] based on the approach of the vibrational mechanics [Blekhman, 2000]. It was experimentally confirmed that the inhomogeneity of the vibration field and the more complex trajectories of motion of various points of the platform, primarily in the screens, make it possible to obtain an appreciable technological effect [Blekhman and Vaisberg, 2011].

In [Chelomey, 1978-1981], the problem of constructing a plane-parallel field of trajectories of the points of the platform of a one-rotor vibrational machine is solved by means of an eccentric arrangement of the vibrator. In the simplest case, when the vibrator is positioned at the center of mass, the platform performs plane-parallel circular translational oscillations and the trajectory of any of its points in the vertical plane is described by the equation of circles having the same parameters, that is, the created field of vibrations is homogeneous. With the eccentric location of the actuator, the points of the platform oscillate along trajectories close to elliptical ones, and the ratios and absolute sizes of the axes of the ellipses differ for different points, that is, the field of vibrations is inhomogeneous.

In [Blekhman, Vaisberg, and Firsova, 2001; Firsova, 2002(1); Firsova, 2002(2)] the construction of a vibration field for a VU with two actuators is studied. It is shown that it is possible to obtain a more diverse picture of the fields of trajectories in comparison with one-rotor installations. The problem of synthesis and analysis of vibration fields is considered and universal field diagrams are constructed. It is shown that the vibration field of the two-rotor vibration system depends on the coordinates of the points of fastening of the rotors, the mass of the debalances and the steady phase difference of the debalances provided they are in stable synchronous rotation mode.

In [Rumyantsev and Tarasov, 2010] is shown that us- 


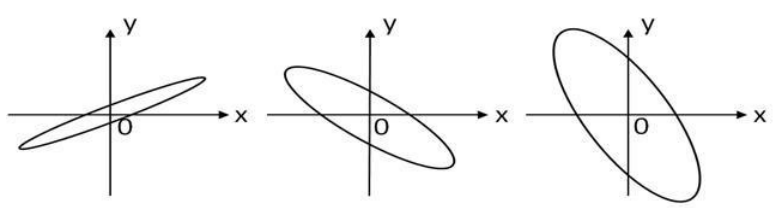

Figure 1. The diagram of the motion of various points of the supporting body for three-rotor machine [Rumyantsev and Tarasov, 2010].

\section{Two-rotor Vibration Unit: Kinematics and Dy- namics}

Efficiency of the proposed algorithms is analyzed for 2-rotor vibration unit model with 6 degrees of freedom taking into account 3 degrees of freedom for supporting body (Fig. 2). Here $\varphi_{1}, \varphi_{2}$ are the rotation angles of

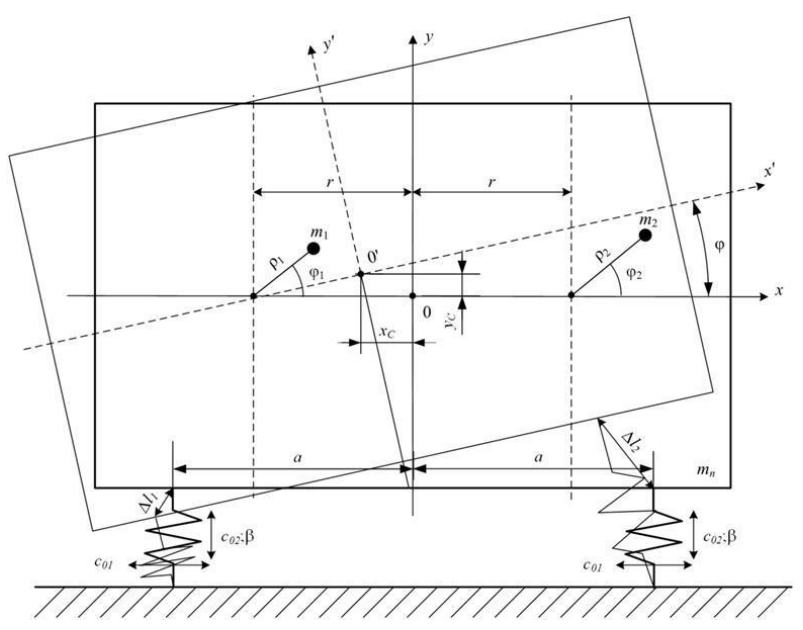

Figure 2. The kinematic scheme of controlled vibrational stand.

the rotors measured from the horizontal position, $x_{c}$, $y_{c}$ are the horizontal and vertical displacement of the supporting body from the equilibrium position, $m_{i}$ are the masses of the rotors, $\varrho_{i}$ is the eccentricity of rotors, $c_{01}, c_{02}$ are the horizontal and vertical spring stiffness, $m_{0}=m_{n}+m_{1}+m_{2}$ is the total mass of the unit, $\beta$ is the damping coefficient, $\pm r$ are abscissas of the points of attachment of the rotor axes.

We will assume that rotor shafts are orthogonal to the motion of the support [Tomchina and Kudryavtseva, 2005]. The unit dynamics can be described by the La- 
grange 2nd kind equations

$$
\begin{aligned}
& m_{0} \ddot{x}_{c}+\ddot{\varphi}\left[\left(m_{1}-m_{2}\right) r \sin \varphi-m_{1} \varrho_{1} \sin \left(\varphi+\varphi_{1}\right)-\right. \\
& \left.m_{2} \varrho_{2} \sin \left(\varphi+\varphi_{2}\right)\right]-\ddot{\varphi}_{1} m_{1} \varrho_{1} \sin \left(\varphi+\varphi_{1}\right)- \\
& \ddot{\varphi}_{2} m_{2} \varrho_{2} \sin \left(\varphi+\varphi_{2}\right)+\dot{\varphi}^{2}\left[\left(m_{1}-m_{2}\right) r \cos \varphi-\right. \\
& \left.m_{1} \varrho_{1} \cos \left(\varphi+\varphi_{1}\right)-m_{2} \varrho_{2} \cos \left(\varphi+\varphi_{2}\right)\right]- \\
& \dot{\varphi}_{1}^{2} m_{1} \varrho_{1} \cos \left(\varphi+\varphi_{1}\right)+\dot{\varphi}_{2}^{2} m_{2} \varrho_{2} \cos \left(\varphi+\varphi_{2}\right) \\
& 2 \dot{\varphi} \dot{\varphi}_{1} m_{1} \varrho_{1} \cos \left(\varphi+\varphi_{1}\right)- \\
& 2 \dot{\varphi} \dot{\varphi}_{2} m_{2} \varrho_{2} \cos \left(\varphi+\varphi_{2}\right)+2 c_{01} x_{c}+\beta \dot{x}_{c}=0 ; \\
& m_{0} \ddot{y}_{c}+\ddot{\varphi}\left[\left(m_{2}-m_{1}\right) r \cos \varphi+m_{1} \varrho_{1} \cos \left(\varphi+\varphi_{1}\right)+\right. \\
& \left.m_{2} \varrho_{2} \cos \left(\varphi+\varphi_{2}\right)\right]+\ddot{\varphi}_{1} m_{1} \varrho_{1} \cos \left(\varphi+\varphi_{1}\right)+ \\
& \ddot{\varphi}_{2} m_{2} \varrho_{2} \cos \left(\varphi+\varphi_{2}\right)+\dot{\varphi}^{2}\left[\left(m_{1}-m_{2}\right) r \sin \varphi-\right. \\
& \left.m_{1} \varrho_{1} \sin \left(\varphi+\varphi_{1}\right)-m_{2} \varrho_{2} \sin \left(\varphi+\varphi_{2}\right)\right]- \\
& \dot{\varphi}_{1}^{2} m_{1} \varrho_{1} \sin \left(\varphi+\varphi_{1}\right)-\dot{\varphi}_{2}^{2} m_{2} \varrho_{2} \sin \left(\varphi+\varphi_{2}\right)- \\
& 2 \dot{\varphi} \dot{\varphi}_{1} m_{1} \varrho_{1} \sin \left(\varphi+\varphi_{1}\right)- \\
& 2 \dot{\varphi} \dot{\varphi}_{2} m_{2} \varrho_{2} \sin \left(\varphi+\varphi_{2}\right)+m_{o} g+2 c_{01} y_{c}+\beta \dot{y}_{c}=0 ; \\
& \ddot{x}_{c}\left[\left(m_{1}-m_{2}\right) r \sin \varphi-m_{1} \varrho_{1} \sin \left(\varphi+\varphi_{1}\right)-\right. \\
& \left.m_{2} \varrho_{2} \sin \left(\varphi+\varphi_{2}\right)\right]+\ddot{y}_{c}\left[\left(m_{2}-m_{1}\right) r \cos \varphi+\right. \\
& \left.m_{1} \varrho_{1} \cos \left(\varphi+\varphi_{1}\right)+m_{2} \varrho_{2} \cos \left(\varphi+\varphi_{2}\right)\right]+ \\
& \ddot{\varphi}\left(J-2 m_{1} r \varrho_{1} \cos \varphi_{1}+2 m_{2} r \varrho_{2} \cos \varphi_{2}\right)+ \\
& \ddot{\varphi}_{1}\left(J_{1}-m_{1} r \varrho_{1} \cos \varphi_{1}\right)+ \\
& \ddot{\varphi}_{2}\left(J_{2}+m_{2} r \varrho_{2} \cos \varphi_{2}\right)+\dot{\varphi}_{1}^{2} m_{1} r \varrho_{1} \sin \varphi_{1}- \\
& \dot{\varphi}_{2}^{2} m_{2} r \varrho_{2} \sin \varphi_{2}+2 \dot{\varphi} \dot{\varphi}_{1} m_{1} r \varrho_{1} \sin \varphi_{1}- \\
& 2 \dot{\varphi} \dot{\varphi}_{2} m_{2} r \varrho_{2} \sin \varphi_{2}-\left(m_{1}-m_{2}\right) g r \cos \varphi+ \\
& m_{1} g \varrho_{1} \cos \left(\varphi+\varphi_{1}\right)+m_{2} g \varrho_{2} \cos \left(\varphi+\varphi_{2}\right)- \\
& a^{2}\left(c_{01}-c_{02}\right) \sin 2 \varphi+\beta \dot{\varphi}=0 \text {; } \\
& -\ddot{x}_{c} m_{1} \varrho_{1} \sin \left(\varphi+\varphi_{1}\right)+\ddot{y}_{c} m_{1} \varrho_{1} \cos \left(\varphi+\varphi_{1}\right)+ \\
& \ddot{\varphi}\left(J_{1}-m_{1} r \varrho_{1} \cos \varphi_{1}\right)+\ddot{\varphi}_{1} J_{1}- \\
& \dot{\varphi}^{2} m_{1} r \varrho_{1} \sin \varphi_{1}+m_{1} g \varrho_{1} \cos \left(\varphi+\varphi_{1}\right)+k_{c} \dot{\varphi}_{1} M_{1} ; \\
& -\ddot{x}_{c} m_{2} \varrho_{2} \sin \left(\varphi+\varphi_{2}\right)+\ddot{y}_{c} m_{2} \varrho_{2} \cos \left(\varphi+\varphi_{2}\right)+ \\
& \ddot{\varphi}\left(J_{2}+m_{2} r \varrho_{2} \cos \varphi_{2}\right)+\ddot{\varphi}_{2} J_{2}+ \\
& \dot{\varphi}^{2} m_{2} r \varrho_{2} \sin \varphi_{2}+m_{2} g \varrho_{2} \cos \left(\varphi+\varphi_{2}\right)+k_{c} \dot{\varphi}_{2}=M_{2} \text {, }
\end{aligned}
$$

where $J=J_{p l}+J_{1}+J_{2}+\left(m_{1}+m_{2}\right) r^{2}, J_{1}, J_{2}$ are the inertia moments of the rotors, $g$ is the gravitational acceleration, $k_{c}$ is the friction coefficient in the bearings, $M_{1}, M_{2}$ are the motor torques (controlling variables). Kinetic and potential energies $T$ and $\Pi$ are as follows:

$$
\begin{gathered}
T=0.5 m_{0} \dot{x}_{c}^{2}+0.5 m_{0} \dot{y}_{c}^{2}+0.5 J \dot{\varphi}^{2}- \\
r\left[m_{1} \varrho_{1} \cos \varphi_{1}-m_{2} \varrho_{2} \cos \varphi_{2}\right] \dot{\varphi}^{2}+ \\
0.5 J_{1} \dot{\varphi}_{1}^{2}+0.5 J_{2} \dot{\varphi}_{2}^{2}+\left[\left(m_{1}-m_{2}\right) r \sin \varphi-\right. \\
\left.m_{1} \varrho_{1} \sin \left(\varphi+\varphi_{1}\right)-m_{2} \varrho_{2} \sin \left(\varphi+\varphi_{2}\right)\right] \dot{x}_{c} \dot{\varphi}+ \\
{\left[\left(m_{2}-m_{1}\right) r \cos \varphi+m_{1} \varrho_{1} \cos \left(\varphi+\varphi_{1}\right)+\right.} \\
\left.+m_{2} \varrho_{2} \cos \left(\varphi+\varphi_{2}\right)\right] \dot{y}_{c} \dot{\varphi}- \\
-m_{1} \varrho_{1} \sin \left(\varphi+\varphi_{1}\right) \dot{x}_{c} \dot{\varphi}_{1}- \\
-m_{2} \varrho_{2} \sin \left(\varphi+\varphi_{2}\right) \dot{x}_{c} \dot{\varphi}_{2}+ \\
+m_{1} \varrho_{1} \cos \left(\varphi+\varphi_{1}\right) \dot{y}_{c} \dot{\varphi}_{1}+ \\
+m_{2} \varrho_{2} \cos \left(\varphi+\varphi_{1}\right) \dot{y}_{c} \dot{\varphi}_{2}+ \\
+\left(J_{1}-m_{1} \varrho_{1} r \cos \varphi_{1}\right) \dot{\varphi}_{\dot{\varphi}}+ \\
+\left(J_{2}+m_{2} \varrho_{2} r \cos \varphi_{2}\right) \dot{\varphi}_{2}, \\
\Pi=m_{0} g y_{c}+\left(m_{2}-m_{1}\right) r g \sin \varphi+ \\
m_{1} \varrho_{1} g \sin \left(\varphi+\varphi_{1}\right)+m_{2} \varrho_{2} g \sin \left(\varphi+\varphi_{2}\right)+ \\
c_{01}\left(x_{c}^{2}+a^{2} \cos ^{2} \varphi\right)+c_{02}\left(y_{c}^{2}+a^{2} \sin ^{2} \varphi\right) \\
H=T+\Pi .
\end{gathered}
$$

The parameter values correspond to the vibration stand SV-2M designed in the IPME [Blekhman, Bortsov, Burmistrov, et. al., 1999; Andrievskii, Blekhman, Blekhman, et.al., 2016].

\section{Integral-differential Speed-gradient Control Al- gorithms for Synchronization of Two-rotor Vi- bration Unit}

Frequency synchronization is defined as an exact coincidence of angular velocities of the unbalanced rotors $\omega_{s}=\omega_{r} ; s, r=1, \ldots, k$. [Blekhman, 2000]. For practice approximate synchronization conditions are more appropriate [Blekhman and Fradkov, 2004]:

$$
\left|\omega_{s}-\omega_{r}\right| \leq \varepsilon,
$$

where $\varepsilon>0$ can be chosen numerically as $\varepsilon>0=$ $0.05 \omega^{*}$, with a given accuracy, similar to conventional transient process measurement. However the ratio (3) may be not sufficient for synchronization, since its fulfillment does not prevent the accumulation of a phase synchronization reduced error (reduced phase shift). That is why there is a need to impose additional requirements on the system phases. To this end the notion of approximate phase synchronization is formulated as follows [Blekhman and Fradkov, 2004]:

$$
\left|\varphi_{s}-\varphi_{r}-L_{s r}\right|<\varepsilon_{1}, \quad s, r=1, \ldots, k .
$$

Equations (3) and (4) should hold for some $\varepsilon_{1}>0$, and some real $L_{s r}$.

To provide a synchronous rotation mode of unbalanced rotors for system (1), it is suggested to use speedgradient method [Andrievskii, Stotskii, Fradkov, 1988; Fradkov, 2007] with the following goal function:

$$
Q(z)=\frac{1-\alpha}{2}\left(H-H^{*}\right)^{2}+\frac{\alpha}{2}\left(\dot{\varphi}_{1} \pm \dot{\varphi}_{2}\right)^{2},
$$

where $0<\alpha<1$ is weight coefficient; $H$ is total mechanical energy of a system (1), $H^{*}$ is the desired value of $H$. The speed-gradient algorithm in the finite form with the objective functional (5) is as follows [Tomchina and Kudryavtseva, 2005]:

$$
\begin{aligned}
& M_{1}=-\gamma_{1}\left\{(1-\alpha)\left(H-H^{*}\right) \dot{\varphi}_{1}+\frac{\alpha}{J_{1}}\left(\dot{\varphi}_{1} \pm \dot{\varphi}_{2}\right)\right. \\
& M_{2}=-\gamma_{2}\left\{(1-\alpha)\left(H-H^{*}\right) \dot{\varphi}_{2} \pm \frac{\alpha}{J_{2}}\left(\dot{\varphi}_{1} \pm \dot{\varphi}_{2}\right)\right\}
\end{aligned}
$$

For our purpose the proportional-integral (PI-) control 
algorithm is better suited

$$
\begin{gathered}
M_{1}=-\gamma_{1}\left\{( 1 - \alpha ) \left[\left(H-H^{*}\right) \dot{\varphi}_{1}+\right.\right. \\
\left.\int\left(H-H^{*}\right) \dot{\varphi}_{1} d t\right]+\frac{\alpha}{J_{1}}\left(\dot{\varphi}_{1} \pm \dot{\varphi}_{2}\right)+ \\
\left.\frac{\alpha}{J_{1}}\left(\dot{\varphi}_{1} \pm \dot{\varphi}_{2}+C_{1}\right)\right\} ; \\
M_{2}=-\gamma_{2}\left\{( 1 - \alpha ) \left[\left(H-H^{*}\right) \dot{\varphi}_{2}+\right.\right. \\
\left.\int\left(H-H^{*}\right) \dot{\varphi}_{2} d t\right] \pm \frac{\alpha}{J_{2}}\left(\dot{\varphi}_{1} \pm \dot{\varphi}_{2}\right) \pm \\
\left.\frac{\alpha}{J_{2}}\left(\dot{\varphi}_{1} \pm \dot{\varphi}_{2}+C_{2}\right)\right\} ;
\end{gathered}
$$

where $C_{1}, C_{2}$ are some constant phase shifts. To simplify the implementation of a PI-algorithm, the integrals in the first terms in right hand parts of (7) may be omitted

$$
\begin{gathered}
M_{1}=-\gamma_{1}\left\{(1-\alpha)\left(H-H^{*}\right) \dot{\varphi}_{1}+\frac{\alpha}{J_{1}}\left(\dot{\varphi}_{1} \pm \dot{\varphi}_{2}\right)+\right. \\
\left.\frac{\alpha}{J_{1}}\left(\dot{\varphi}_{1} \pm \dot{\varphi}_{2}+\Delta \varphi_{1}^{*}\right)\right\} ; \\
M_{2}=-\gamma_{2}\left\{(1-\alpha)\left(H-H^{*}\right) \dot{\varphi}_{2} \pm \frac{\alpha}{J_{2}}\left(\dot{\varphi}_{1} \pm \dot{\varphi}_{2}\right) \pm\right. \\
\left.\frac{\alpha}{J_{2}}\left(\dot{\varphi}_{1} \pm \dot{\varphi}_{2}+\Delta \varphi_{2}^{*}\right)\right\} ;
\end{gathered}
$$

The accuracy of such a simplification is reasonable after the transient process, i.e. in the steady-state mode. The choice of the values $\Delta \varphi_{i}^{*}$ in the term $\left(\dot{\varphi}_{1} \pm \dot{\varphi}_{2}+\right.$ $\left.\Delta \varphi_{i}^{*}\right)$, allows one to specify the value of the reduced phase shift $\Delta \varphi(\infty)=\varphi_{1}-\varphi_{2}$. In this paper the choice $\Delta \varphi_{2}^{*}=0$ is proposed.

\section{Simulation Results}

Simulation results are obtained using the MATLAB environment. The first set of the results are related to the case of synchronization with algorithm (8) for the case of identical rotors $m 1=m 2=1.5 \mathrm{~kg}$ and changing $\Delta \varphi_{1}^{*}$ in Fig. 3. Plots a) exhibit the phase shift $\Delta \varphi=\varphi_{1}-\varphi_{2}$; plots b) show angular velocity of the rotors. Diagrams of the supporting body vibration field sampled in 5 different points are shown in Fig.4. The left oval corresponds to the loading end while the right oval corresponds to the deployment end. The left plot of Fig. 3 a) is obtained for $\Delta \varphi=-1.1 \mathrm{rad}$ while the right pictures correspond to the case $\Delta \varphi=0.52$ $\mathrm{rad}$. As is seen from the plots the phase synchronization takes place: $\Delta \varphi(\infty) \rightarrow L_{12}=$ const and the vibration fields are inhomogeneous.

In practice the rotors of vibroactuators are usually nonidentical. Therefore it is important to study VU dynamics for nonidentical rotors. Let $m_{1}=1.5 \mathrm{~kg}$, $m_{2}=2.5 \mathrm{~kg}$. Simulation results for conventional control (for constant torques $M_{1}, M_{2}$ ) are shown in Fig. 5, left and in Fig. 6, top. In Fig. 5, left phase difference and angular velocities are shown while in Fig. 6, top the trajectories of the five points located on the horizontal line crossing the center of mass of the platform are depicted. As is seen from the plots, the frequencies of the rotors' rotation are different $\left(\omega_{1}(t) \neq \omega_{2}(t)\right)$ and $\varphi_{1}(t)-\varphi_{2}(t) \rightarrow \infty$, i.e. synchronization fails. It leads to faster equipment wear and degradation of the technological process performance.

In Fig. 5, right and in Fig.6, bottom the simulation results for the VU controlled by the algorithm (8) are shown. The VU and control algorithm parameters are as follows: $H^{*}=500 \mathrm{~J}, m_{1}=1.5 \mathrm{~kg}, m_{2}=2.5$ $\mathrm{kg}, \Delta \varphi_{1}^{*}=-2 \mathrm{rad} ; \Delta \varphi_{2}^{*}=0 \mathrm{rad} ; i=1,2$. It is seen that the VU controlled by the algorithm (8) has better efficiency of vibration transportation: the loading of the raw material from the bunker to the left end is more efficient if the direction of the big semiaxis of the left ellipse is closer to the horizontal one. Moving the material from the left to the right will get the above semiaxis more and more vertical while the amplitude of the vertical vibrations will be growing (note that the right rotor has bigger mass than the left one). Therefore screening at the right end of the platform will be more intense. Additionally it improves the screening process as a whole and reduces congestion during unloading.

Simulation results suggest that in the case of nonidentical rotors the proposed control algorithm ensures the synchronous mode even when the conventional control with constant torques does not guarantee selfsynchronization. Thus, the developed algorithm for controlling synchronization of rotors allows one to synthesize various vibrational fields by varying the reduced phase shift $\Delta \varphi_{1}^{*}$. Specifying the $\Delta \varphi_{1}^{*}$ value in the algorithm (8) allows one to specify the steady-state value of the phase shift $\Delta \varphi(\infty)$, which determines the inclination of the elliptical trajectories, optimizing the work of the VU for specific technological conditions and processes.

As established in [Firsova, 2002(2)], an analysis of the equations describing the oscillations of each point of the platform shows that with a fixed mass $m_{p l}$ and the moment of inertia of platform $J$, the oscillation field of the platform is uniquely determined by setting the following seven parameters:

$$
\begin{gathered}
A_{1}=m_{1} \varrho_{1} / m_{p l}, A_{2}=m_{2} \varrho_{2} / m_{p l}, \\
\Delta \varphi=\varphi_{1}-\varphi_{2}, x_{1}, x_{2}, y_{1} y_{2},
\end{gathered}
$$

where $m_{p l}$ is the mass of the platform, $m_{1}, \varrho_{1}, m_{2}, \varrho_{2}$ are mass and the excentricity of the rotors, $x_{1}, x_{2}, y_{1}, y_{2}$ are coordinates of the vibroactuator shafts in the frame associated with the platform with the origin in its center of mass. Thus, with unchanged mass-inertial parameters and fixed coordinates of the attachment points of vibration exciters of a specific vibration installation, the field of vibrations determines the steady-state phase shift of the rotors $\Delta \varphi$. 

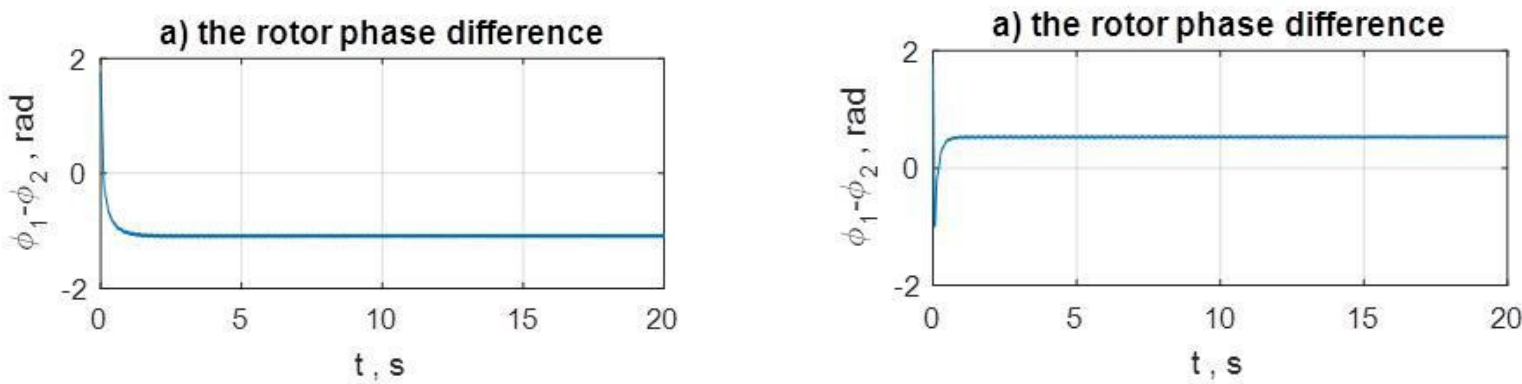

b) angular velocities

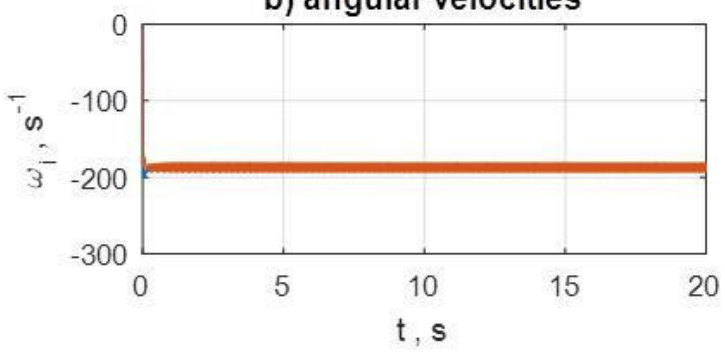

b) angular velocities

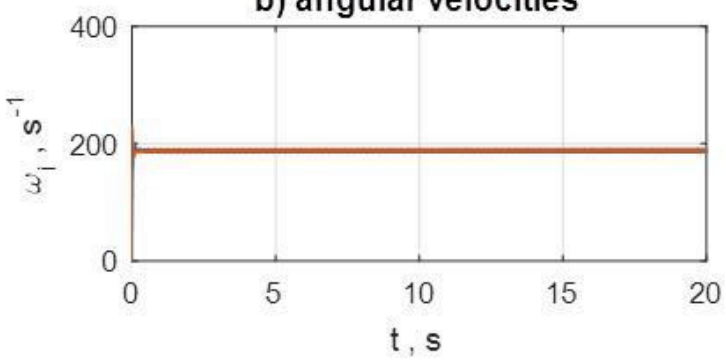

Figure 3. Phase differences and angular velocities. Left: $\Delta \varphi=-1.1 \mathrm{rad}$, right: $\Delta \varphi=0.52 \mathrm{rad}$.
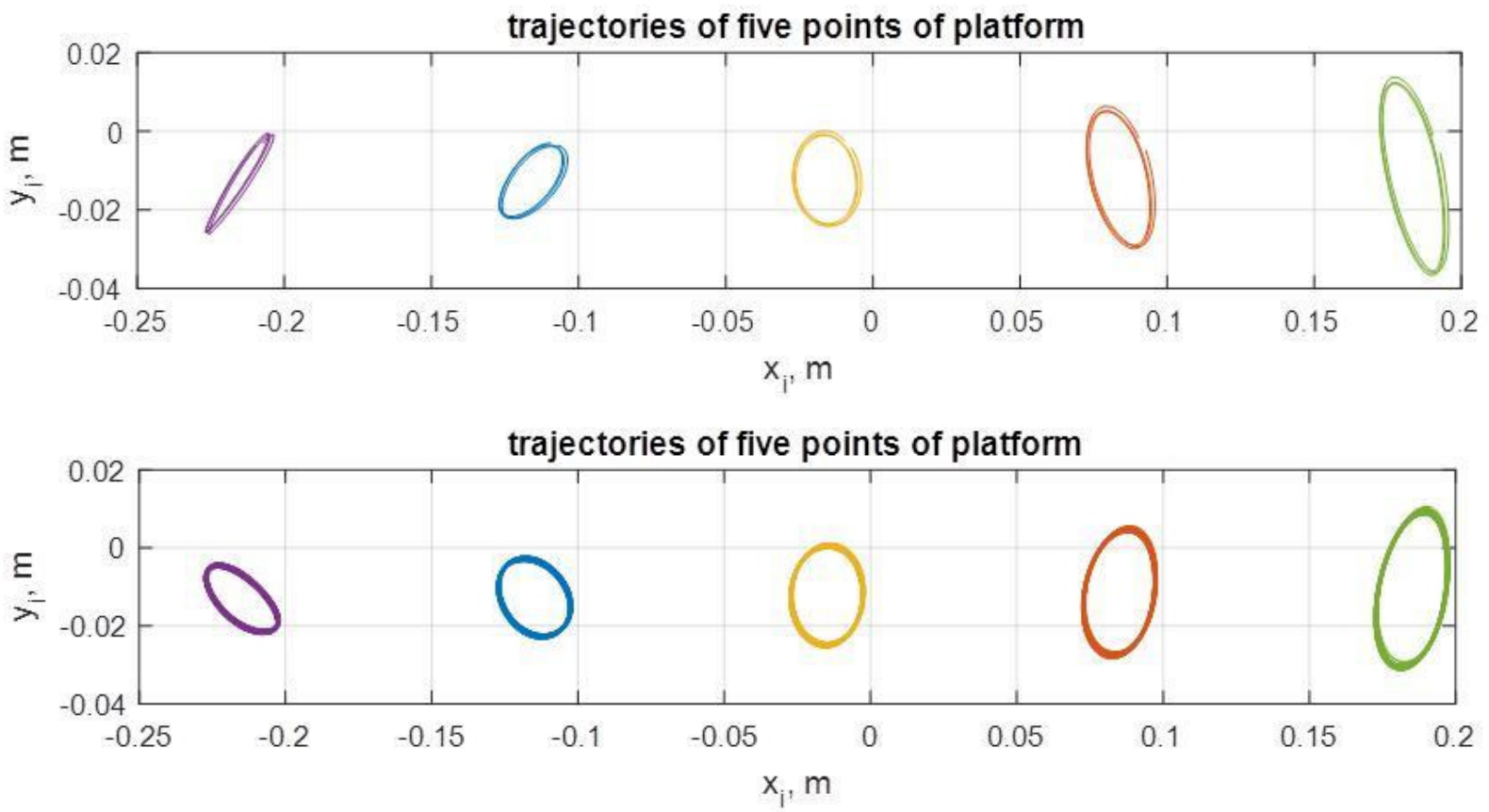

Figure 4. Vibration fields. Top: $\Delta \varphi=-1.1 \mathrm{rad}$, bottom: $\Delta \varphi=0.52 \mathrm{rad}$. 


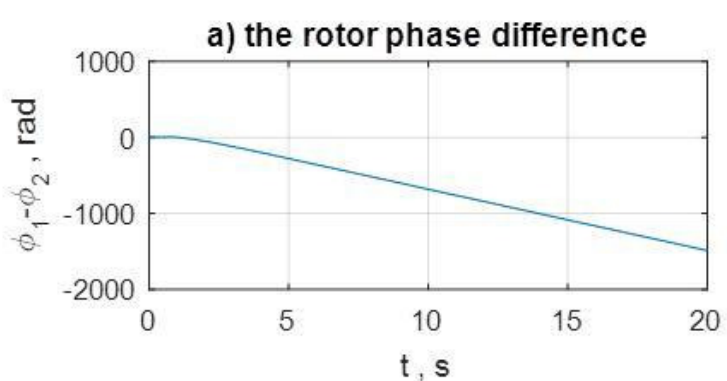

b) angular velocities

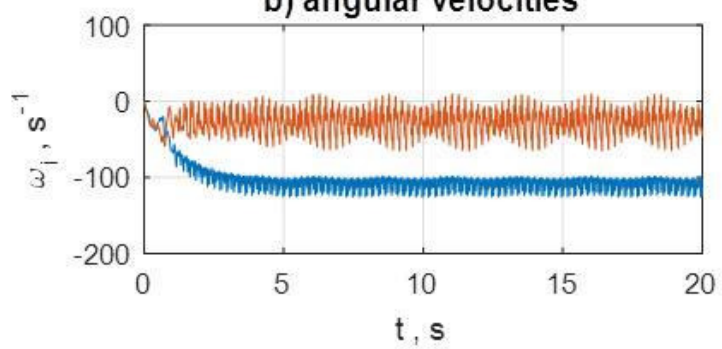

a) the rotor phase difference

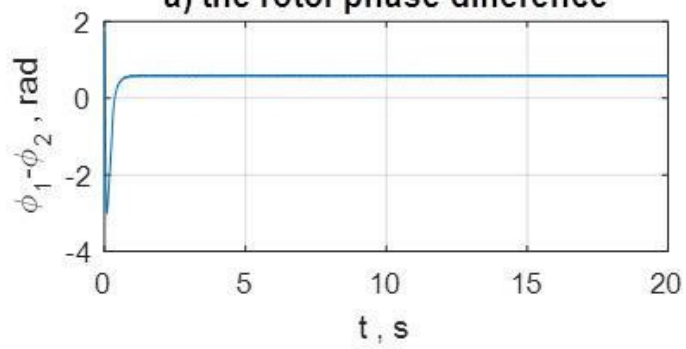

b) angular velocities

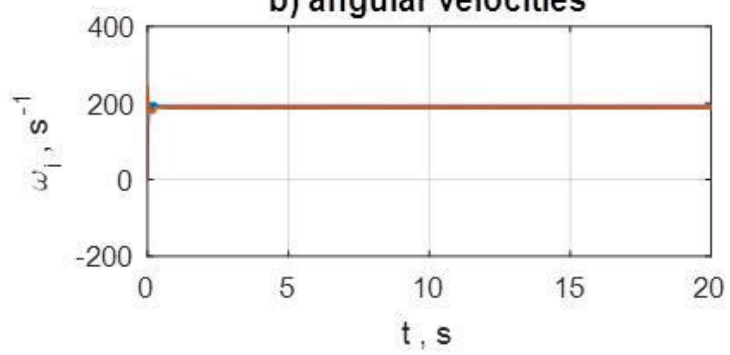

Figure 5. Phase differences and angular velocities for nonidentical rotors. Left: without control (constant torques), right: with control.
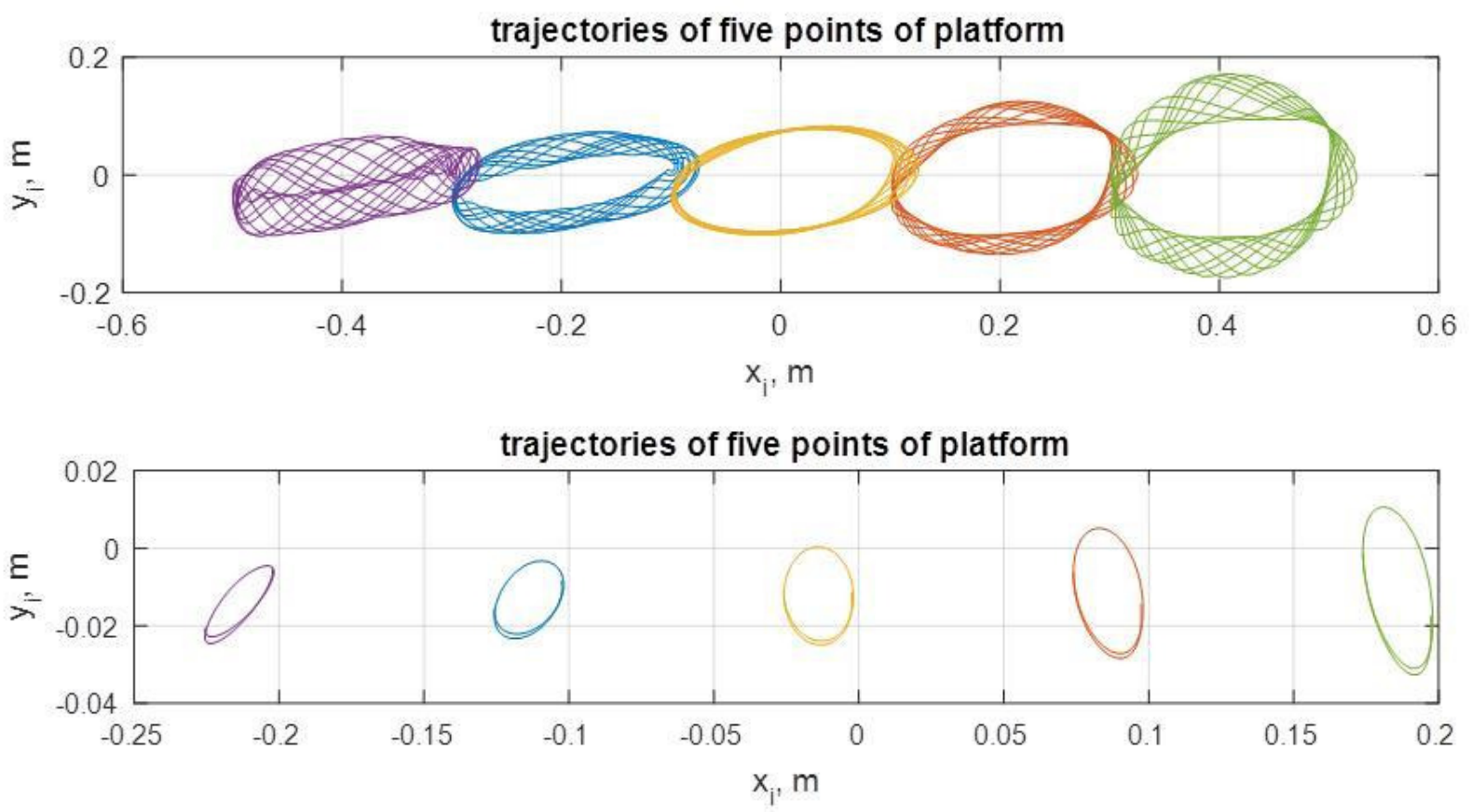

Figure 6. Vibration fields for nonidentical rotors. Top: without control (constant torques), bottom: with control. 


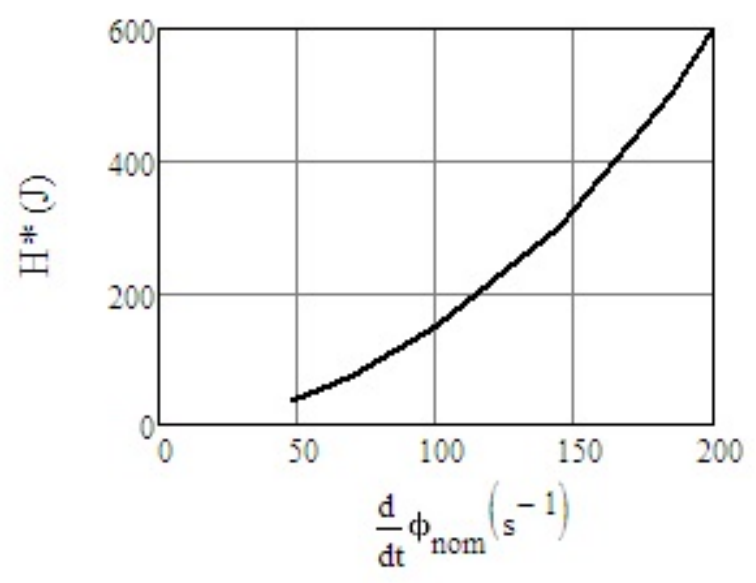

Figure 7. Evaluation of the desired value $H^{*}$

To control the synchronous rotation mode of the rotors using the algorithm (8) it is necessary to set two parameters $H^{*}$ and $\Delta \varphi_{1}^{*}$ in the algorithm. As a rule, when the vibration equipment is in operation, the desired nominal speed of rotation of the rotors, i.e. nominal angular velocity $\dot{\varphi}_{n o m}$. Then the desired value $H^{*}$, appearing in the algorithm, can be calculated for a specific vibration installation according to the diagram of Fig.7. Note that the value of the total energy $H^{*}$ differs significantly from the energy of the rotors themselves due to the significant mass of the supporting body (platform), which receives its energy only thanks to the rotors.

In Fig. 8 the nomograms which determine the dependence of the steady-state value of the limit phase difference of the rotors $\Delta \varphi$ on the value of the phase difference $\Delta \varphi_{1}^{*}$ specified in the synchronization control algorithm (8) are presented. Nomograms were obtained for the vibration stand SV-2M model using intensive computer simulation. Each curve of the nomogram corresponds to a certain set of the rotation speed of the rotors, by which the value of $H^{*}$ is determined. Thus, the desired form of the vibration field can be determined using simulation for one fixed rotor speed $\varphi_{i}$, and in case of changing the given speeds, the required $\Delta \varphi_{1}^{*}$ value appearing in the algorithm (8) is determined by nomogram in Fig.8.

\section{Conclusion}

The problem of feedback control of vibrational fields in a vibration unit is posed. The algorithms for control of the vibration fields by means of the control of the phase shift between rotors based on the speedgradient method [Fradkov, 2007] are proposed. The performance of the closed loop system is examined by simulation for the two-rotor vibration unit SV-2M. It is shown that control allows for new possibilities of control of vibration in the whole supporting body of a vibration machine. It also allows to reduce dependence of

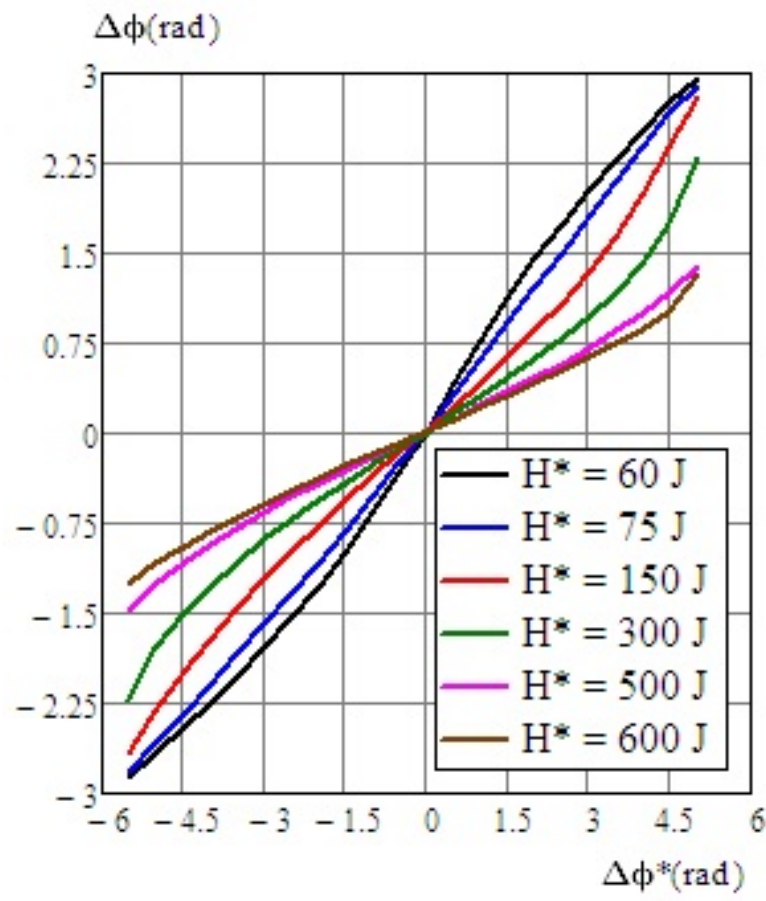

Figure 8. Nomogram representing the dependence of the desired limit phase shift $\Delta \varphi$ on the control algorithm parameter $\Delta \varphi_{1}^{*}$.

the desired mode on the disturbances and on inhomogeneity of the rotors. Finally the proposed nomograms provide a possibility to determine the desired form of the vibration field using simulation for one fixed rotor speed.

\section{Acknowledgements}

The author takes a chance to thank Dr. Dmitry Gorlatov for his assistance with the presentation of the paper.

\section{References}

Andrievskii, B.R., Blekhman, I.I., Blekhman, L I., Boikov, V.I., Vasil'kov, V.B., and Fradkov, A.L. (2016). Education and research mechatronic complex for studying vibration devices and processes. $J$. Mach. Manuf. Reliab., 45(4), pp. 369-374.

Andrievsky, B. and Boikov, V. (2017). Experimental study of multiresonance mechatronic vibrational laboratory set-up. Cybernetics And Physics, Vol. 6, No. 1, pp. 5-12.

Andrievskii, B.R., Stotskii, A.A., Fradkov, A.L. Velocity-Gradient Algorithms In Control And Adaptation Problems. Automation And Remote Control. Vol. 49, Is. 12, pp. 1533-1564, Part: 1, 1988.

Blekhman, I.I.(2000). Vibrational Mechanics: Nonlinear Dynamic Effects, General Approach, Applications. World Scientific.

Blekhman, I.I., Bortsov, Y.A., Burmistrov, A.A., Fradkov, A.L., Gavrilov, S.V., Kononov, O.A., Lavrov, 
B.P., Sokolov, P.V., Shestakov, V.M., and Tomchina, O.P. (1999). Computer-controlled vibrational setup for education and research. In Proc. of 14th IFAC World Congress, vol. M, pp. 193-197.

Blekhman, I. and Fradkov, A. L. Eds. (2001). Control of Mechatronic Vibration Units. St. Petersburg: Nauka, (in Russian).

Blekhman, I.I. and Fradkov, A.L. (2004). On general definitions of synchronization. In: Selected topics in vibrational mechanics, Ed. I.I.Blekhman. Singapore: World Scientific, pp. 179-188.

Blekhman, I.I., Vaisberg, L.A., and Firsova, A.D. (2001). Evaluation of the field of the trajectories for the body of the vibration machine with two unbalanced vibroactuators. Obogaschenie Rud, No. 2. pp. 39-42 (In Russian).

Blekhman, I.I. and Vaisberg, L.A. (2011). Selfsynchronization as a selforganization phenomenon and a basis for development of energy efficient technologies. In Proc. 10th Biennial International Conference on Vibration Problems (ICOVP), Prague, Czech Republic, Sep. 05-08, pp. 365-370.

Chelomey, V. L., Ed. (1978-1981). Vibration in Engineering. Moscow: Machinostroenie, vol. 4, (in Russian).

Firsova, A.D. (2002, 1). Stationary oscillations of the operating part of vibration machine with two arbitrary located unbalance vibration exciters. In Book of $A b$ stracts of the Annual Scientific Conference GAMM, Augsburg, p. 44.

Firsova, A.D. (2002, 2). Investigation of vibration field of a regid body set in motion by two synchronously working vibration exciters. In Proc. of XXIX Summer school "Advanced Problems in Mechanics" (APM 2001), IPME RAS, pp.231-235.

Fradkov, A. Cybernetical Physics. From Control of Chaos to Quantum Control. Springer-Verlag, Berlin, Heidelberg, 2007.

Rumyantsev, S. and Tarasov, D. (2010). Numerical Simulation of Nonlinear Dynamics of Vibration Transport Machines in Case of Three Independently Rotating Vibration Exciters. Recent Advances in Applied Mathematics: Proceedings of the American Conference on Applied Mathematics (AMERICANMATH'10). Harvard University, Cambridge, USA, Jan. 27-29, pp. 191-194.

Tomchina, O. and Kudryavtseva, I. (2005). Controlled synchronization of unbalanced rotors with flexible shafts in time-varying vibrational units. 2005 Intern. Conf. Physics and Control, PhysCon 2005, pp. 790794, St. Petersburg; Russia.

Tomchin D.A., Tomchina O.P., Fradkov A.L. Controlled Passage through Resonance for Flexible Vibration Units. Mathematical Problems in Engineering. V. 2015 (2015), Article ID 839105, 8 pages

Zhang, X.L., Wen, B.C., and Zhao, C.Y. (2012). Synchronization of three homodromy coupled exciters in a non-resonant vibrating system of plane motion. Acta Mechanica Sinica, 28(5), pp. 1424-1435. 